\title{
Hydatid disease of the parotid gland - a case report
}

\author{
Dr. Dharm Chand Kothari ${ }^{1}$, Dr. Shashi Bhushan Tailor ${ }^{2}$, \\ Dr. Harsh Kumar Baid ${ }^{3}$, Dr. Prakash Singh ${ }^{4}$ \\ 1,2,3 (Department of pathology, Sardar Patel Medical College, Bikaner, India) \\ ${ }^{4}$ (Department of pathology, Swai Man Singh Medical College, Jaipur, India)
}

\begin{abstract}
Hydatid disease is a parasitic infestation by a tapeworm of the genus Echinococcus. Hydatid disease most commonly involves the liver and lungs; the abdominal cavity organs stand next in frequency, the parotid gland is a rare site of this disease. The disease is prevalent in most part of the world, though it is most extensive in the sheep and cattle raising areas. Human echinococcosis is a zoonotic infection caused by the tapeworm of the genus Echinococcus and transmitted by dog and other canine animals. It is a serious problem in tropical areas and is seen in many parts of India. In children lungs are the most common site of infection, whereas in adults liver is infected most frequently. Primary hydatid cyst of the parotid gland is extremely rare, even in the endemic areas and very few cases are reported in parotid gland.

Case report: A 23 year old male patient was presented with painless swelling of the right parotid gland. He was treated initially with antibiotics with no improvement. The microscopic examination of FNA aspirated material corresponded to a cystic inflammatory process. The parotid tumor was surgically removed. Pathological study of the cystic lesion revealed hydatid disease of the parotid gland.
\end{abstract}

Keywords: Echinococcus granulosus, Hydatid cyst, Parotid

\section{Introduction}

Hydatid disease most commonly involves the liver and lungs; the abdominal cavity organs stand next in frequency. ${ }^{4}$ The parotid gland is a rare site of this disease. ${ }^{1,2,3,4}$ The parasite has dog- sheep cycle, but man becomes an accidental intermediate host.

Hydatid disease in people is mainly caused by infection with the larval stage of the dog tapeworm Echinococcus granulosus. It is an important pathogenic, zoonotic and parasitic infection (acquired from animals) of humans, following ingestion of tapeworm eggs excreted in the faeces of infected dogs. Hydatid disease is a major endemic health problem in certain areas of the world. It is more a disease of temperate climates than of tropical areas.

Habitat - man (intermediate host) harbors the larval form and not the adult worm, which is however found in the small intestine of dogs (definitive host) and other canine animal. ${ }^{6}$

Morphology - Adult worm - it is small tapeworm measuring 3-6mm in length. It is consists of a scolex, a short neck, and the trunk composed of only three proglottides, the anterior immature, middle mature and posterior gravid. ${ }^{6}$

Egg - 32- 36 $\mu$ in length and $25-32 \mu$ in breath and contains a hexacanth embryo with three pair of hooks. The egg is infective to man, cattle, sheep, and herbivorous animals ${ }^{6}$.

The egg discharged by the definitive host when ingested by an intermediate host, the hexacanth embryos are hatched out, and the embryo bore their way through intestinal wall and enter to the liver through portal circulation and to be arrested in the sinusoidal capillaries. The liver act as the first filter. Some of the embryos may enter into the pulmonary capillaries and lung act as second filter. A few of the embryo may pass the pulmonary capillaries, enter in the general blood stream and lodge in various organs. So embryo may lodge in the parotid gland in this way in rare circumstances ${ }^{6}$.

Wherever the embryo settles, it forms a hydatid cyst, the young larva being transformed in to a hollow bladder. From the inner side of the cyst brood capsule with a number of scolices are developed. A fully developed scolex is a product and its presence inside the hydatid cyst is a sign of "a complete biological development. ${ }^{6}$

\section{Case Report}

A 23 years old, Hindu male was presented to the surgical OPD with the complaint of a gradually increasing painless swelling in the right parotid region for 1 year duration. The swelling was painful to start with but later became painless. Physical examination revealed a cystic swelling, $7 \mathrm{~cm}$ x $5.5 \mathrm{~cm}$-in size, in the parotid region. It was non-tender and mobile. No symptom of facial nerve involvement was recorded. A provisional clinical diagnosis of a cyst or a neoplasm of the parotid gland was made. There was no other palpable lump or swelling anywhere in the body. 
On ultrasonography a cystic mass was reported.

Computed tomography (CT) scan of the head and neck revealed a round, well-demarcated water-density mass in the right parotid gland.

Fine needle aspiration of a parotid mass revealed inflammatory process with cystic changes.

Hematological investigation- Haemoglobin-14.5gm\%, Total Leukocyte Count $-9500 / \mathrm{mm}^{3}$, ESR $-20 \mathrm{~mm}$ at the end of one hour. Bleeding time and clotting time were within normal limit.

Urine microscopy and biochemistry was normal.

Superficial parotidectomy was carried out under general anesthesia.

At operation, a cystic mass was found replacing most of the parotid gland; the parotid capsule was blended with the fibrous wall of the cystic mass. The cystic mass and the remains of the parotid gland and its capsule were excised.

On gross examination cystic mass shows a capsule. On cut section there is a rim of parotid tissue seen on one side of the cyst. The inner wall of cyst is smooth, pale yellow in colour and shining.

Multiple section from cyst was taken, processed and stain with hematoxyline and eosin and examined under microscope. Histopathlogical findings confirm the diagnosis.

Section shows parotid gland parenchyma with hydatid cyst (fig-2) composed of chitinous laminated wall (ectocyst), endocyst having brood capsule and scolices (fig-1). The outer pericyst consists of parotid gland parenchyma and fibrous tissue. Laminated membrane (ectocyst) (fig-3) an elastic covering is present outside to endocyst. Innermost layer (endocyst) is a single layer of cells lining the inner aspects of the cyst and is the only living component, being responsible for the formation of the other layers as well as the hydatid fluid and brood capsules within the cyst and shows scolex from parotid hydatid cyst with hooklets and suckers invginated in a vasicles (fig-4).

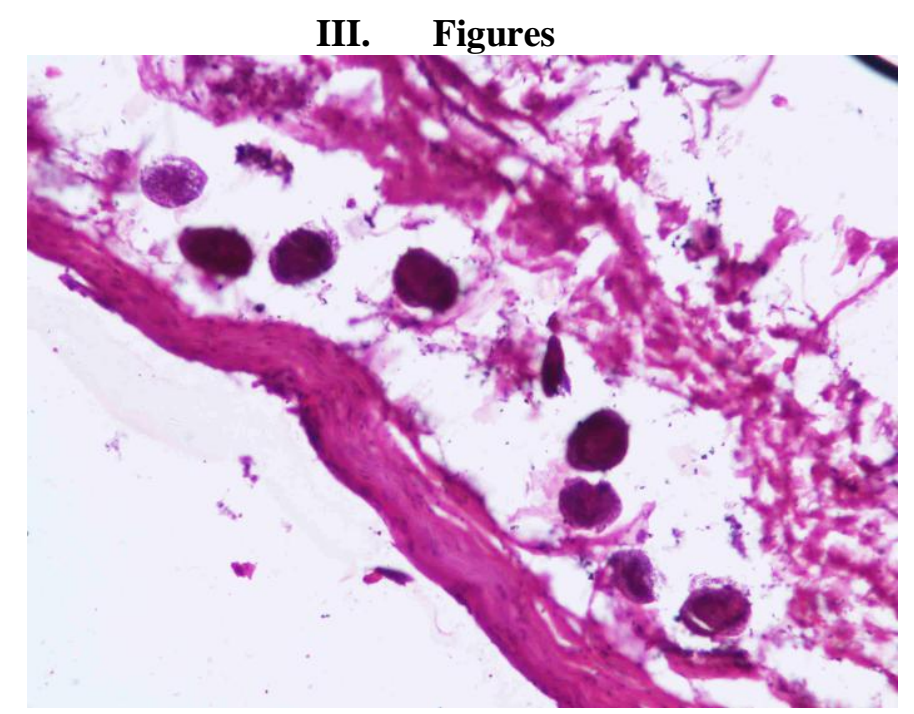

Figure 1 - Hydatid cyst of parotid gland showing pericyst, ectocyst endocyst and few brood capsules.

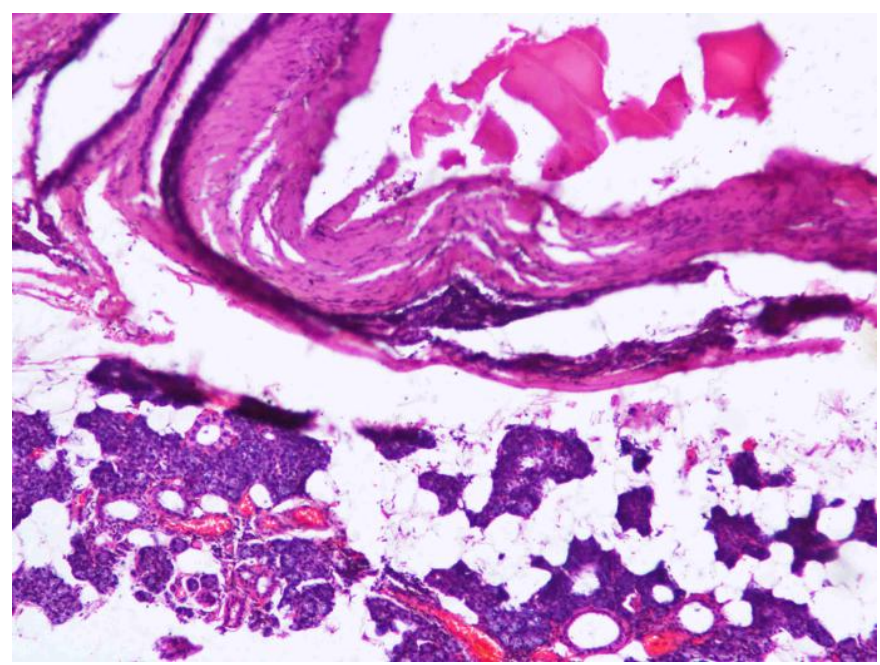

Figure -2 showing chitinous wall of cyst and at the periphery of cyst parotid gland parenchyma. 


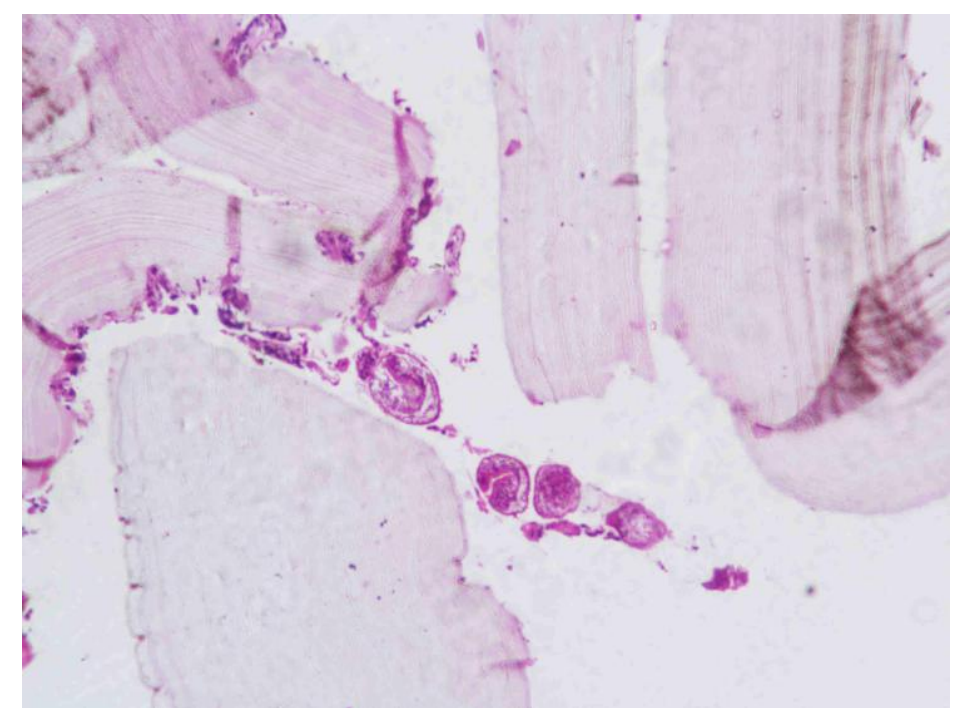

Figure 3 - shows laminated hyaline membrane of (ectocyst) and inner granular germinal and free brood capsules.

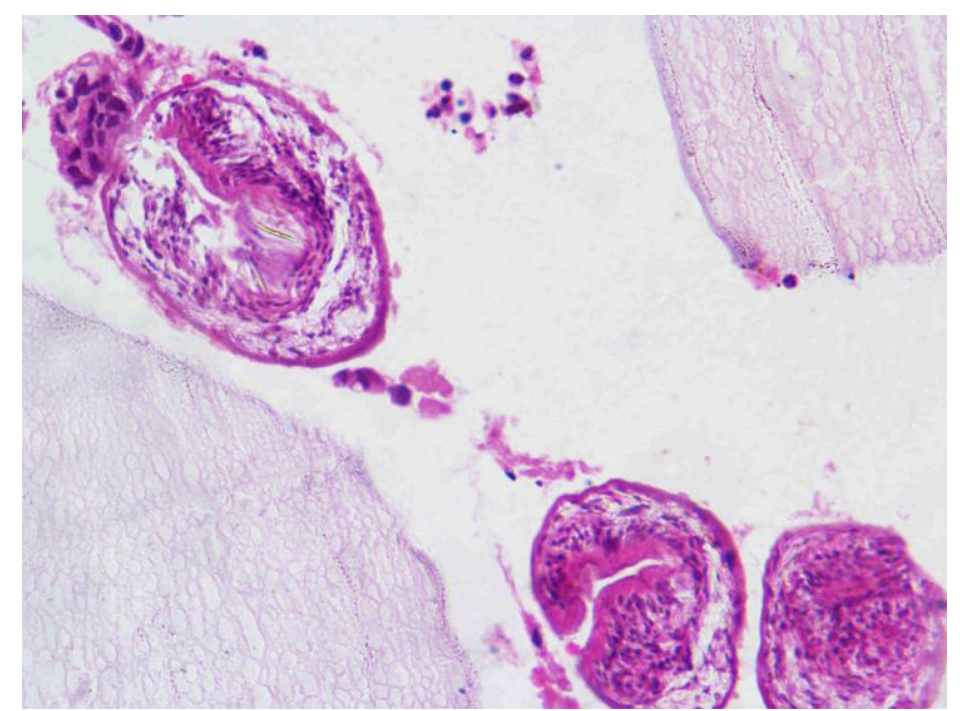

Figure -4 shows scolex from parotid hydatid cyst with hooklets and suckers invginated in a vasicles.

\section{Discussion}

Hydatid disease in people is mainly caused by infection with the larval stage of the dog tapeworm Echinococcus granulosus. It is an important pathogenic, zoonotic and parasitic infestation (acquired from animals) of humans caused by ingestion of tapeworm eggs excreted in the faeces of infected dogs. Hydatid disease is a major endemic health problem in certain areas of the world.

Cystic hydatid disease usually affects the liver (50-70\%) and less frequently the lung, the spleen, the kidney, the bones, and the brain.

The parotid gland hydatid cysts are always primary. A hexacanth embryo, having a size of about 30 to $35 \mu \mathrm{m}$ may unusually cross the liver and lung capillary beds; and once in arterial circulation, it may lodge itself in any site of the body, mostly the kidney, brain, bone, spleen and the heart, and rarely the parotid gland. This should be kept in mind while interpreting a cystic or soft tumour-like mass affecting the gland, because the accidental rupture of the cyst during surgical intervention may cause life threatening complications to the patient due to an anaphylaxis and the locally spilled hydatid material may results in secondary cyst formation, so the hydatid cyst must be removed with capsule without puncture.

\section{Conclusion}

Hydatid cyst of the parotid gland is very rare. Where the incidence of the disease is high, hydatid cyst of parotid gland should be considered in the differential diagnosis of lesions causing swelling of the parotid area. 


\section{References}

[1]. Amir-Jahed, A. K., Fardin, R. and Bakshandeh, K.: Clinical echinococcosis. Ann. Surg., 182: 541-546, 1975.

[2]. Azizi, D.: Remarks on the compiled cases of hydatid cyst operated on in Tehran. Sci. J. Iranian Med. Council, 2: 127. Cited by Emamy and Asadian.

[3]. Saxena S K, Chaudhary S K, Saxena G R, Rao S S. Hydatid cyst of the parotid gland (a case report). J Postgrad Med 1983;29:105

[4]. Darabi M, Varedi P, Mohebi AR, Mahmoodi S, Varedi P, Nabavizadeh SA, Erfan A, Ostadali Makhmalbaf A, Saedi D, Saadat Mostafavi SR, Mousavi SM. Hydatid cyst of the parotid gland. Oral Maxillofac Surg. 2009 Mar;13(1):33-5.

[5]. $\quad$ Saidi, F.: "Surgery of Hydatid Disease", 1st Ed., W. B. Saunders Co. Ltd., London, Philadelphia and Toronto, 1976, p. 282

[6]. K.D. Chatterjee. "Parasitology - protozoology and helminthology" $12^{\text {th }}$ edition, published 1980, Calcutta, 1995, p.121-126 